\title{
AN APPLICATION OF THE RIGIDITY OF DOLBEAULT-TYPE OPERATORS
}

\author{
PING LI
}

\begin{abstract}
Suppose a compact, almost-complex manifold admits a compatible circle action. By using the rigidity property and the coefficient of $y+1$ in the $\chi_{y}$-genus simultaneously, we show that the weights on the fixed point set of this circle action have intimate relations, which improves a previous result of the author.
\end{abstract}

\section{Introduction and main result}

In a previous paper [5], the author noticed that some Dolbeault-type operators on compact, almost-complex manifolds are rigid, which means that the equivariant indices of these operators have the property of invariance under compatible circle actions. In particular, if some circle action on a compact, almost-complex manifold has isolated fixed points, this rigidity phenomenon provides us with intimate relations between the weights on the fixed points and the indices of these Dolbeault-type operators [5, Theorem 2.2]. Using this observation, the author gives some applications to symplectic geometry and related topics. Among the others, the author proved a result [5, Theorem 3.5], which generalizes a result of Pelayo and Tolman [9, Theorem 2].

Theorem 3.5 in [5] says that, if a compact, almost-complex manifold admits a compatible circle action with isolated fixed points, then the weights of all the fixed points, when gathering together, can be paired such that the sum of each pair is zero. The original proof of this result in [5] heavily relies on the property that the fixed points are isolated. So in this sense, it cannot be generalized to the general case. Recently, the author found out an alternative proof of this result by combining the rigidity of Dolbeault-type operators and the coefficient of $y+1$ in the Taylor expansion of the Hirzebruch $\chi_{y}$-genus. More details can be found in Section 2. Using this new method, we can generalize [5, Theorem 3.5] to arbitrary fixed point set.

The rest of this section is to fix some symbols and state our main result (Theorem 1.1), whose proof will be given in Section 2. In Section 3 we will explain the motivation of the idea of our proof.

Let $\left(M^{2 n}, J\right)$ be a compact, almost-complex manifold with an almost-complex structure $J$ and real dimension $2 n$. We call a circle action on $\left(M^{2 n}, J\right)$ compatible if it preserves this almost-complex structure $J$. Suppose we have a non-trivial compatible circle action $\left(S^{1}\right.$-action) on $\left(M^{2 n}, J\right)$ with non-empty fixed points:

$$
\varphi: S^{1} \times M \rightarrow M \text {. }
$$

Received by the editors April 2, 2012.

2000 Mathematics Subject Classification. 37B05, 58J20, 32Q60.

Key words and phrases. rigidity, Dolbeault-type operator, circle action, weight. 
Let $\operatorname{Fix}(M)$ be the fixed point set of this action $\varphi$. As is well-known, each connected component of $\operatorname{Fix}(M)$ is a compact, connected, almost-complex submanifold of $M$. Let $F$ be any such a connected component with complex dimension $r(0 \leq r \leq n-1)$. Here $r$ depends on the choice of $F$. Then the normal bundle of $F$ in $M$, which is denoted by $\nu(F)$, can be decomposed into a sum of $n-r$ complex line bundles with respect to this action

$$
\nu(F)=\bigoplus_{i=1}^{n-r} \mathrm{~L}\left(F, k_{i}\right), \quad k_{i} \in \mathbb{Z}-\{0\},
$$

such that for each $v \in \mathrm{L}\left(F, k_{i}\right)$ and $g \in S^{1} \subset \mathbb{C}$, we have

$$
\varphi_{*}(g) \cdot v=g^{k_{i}} \cdot v .
$$

Note that these non-zero integers $k_{1}, \ldots, k_{n-r}$ are not necessarily distinct and depend on the choice of $F$, which we call the weights of this circle action on the connected component $F$.

From now on we use $e(\cdot)$ to denote the Euler number of a manifold. Now we associate each component $F$ in $\operatorname{Fix}(M)(\operatorname{resp} . \operatorname{Fix}(M))$ to a set $\mathrm{S}(F)(\operatorname{resp} . \mathrm{S}(M, \varphi))$ as follows.

$$
\mathrm{S}(F):= \begin{cases}\emptyset, & \text { if } e(F)=0, \\ \bigsqcup_{e(F) \text { copies }}\left\{k_{1}, \ldots, k_{n-r}\right\}, & \text { if } e(F)>0, \\ \coprod_{-e(F) \text { copies }}\left\{-k_{1}, \ldots,-k_{n-r}\right\}, & \text { if } e(F)<0,\end{cases}
$$

and

$$
\mathrm{S}(M, \varphi):=\coprod_{F} \mathrm{~S}(F),
$$

where the sum is over all the connected components in $\operatorname{Fix}(M)$.

Here "Џ" means disjoint union. Although we write $\left\{k_{1}, \ldots, k_{n-r}\right\}$ as a set, repeated elements in it may not be discarded.

Now we can state our main result in this note, which shows that the weights on the components of $\operatorname{Fix}(M)$ whose Euler numbers are non-zero have intimate relations.

Theorem 1.1. Let the notation be as above. Then for any $m \in \mathbb{Z}-\{0\}$, the times $m$ appears in $S(M, \varphi)$ is the same as that of $-m$.

Remark 1.2. (1) This result says nothing about the weights on those connected components whose Euler numbers are zero.

(2) When $\operatorname{Fix}(M, \varphi)$ consists of isolated fixed points, Theorem 1.1 reduces to ([5], Theorem 3.5) as $e(\{\mathrm{pt}\})=1$.

The following corollary is a generalization of [9, Theorem 2] from isolated fixed points to arbitrary fixed points set.

Corollary 1.3. Let the notation be as above. Then

$$
\sum_{F}\left[e(F) \cdot \sum_{i=1}^{n-r} k_{i}\right]=0,
$$

where the sum is also taken over all the connected components in Fix $(M)$.

Let us end this section by using a typical example to illustrate Theorem 1.1. 
Example 1.4. Let $\mathbb{C} P^{n}$ be an $n$-dimensional complex projective space with homogeneous coordinate $\left[z_{0}, z_{1}, \ldots, z_{n}\right]$. Let $\lambda_{1}, \ldots, \lambda_{s}(s \leq n+1)$ be $s$ distinct integers. We can choose $s$ non-negative integers $n_{1}, \ldots, n_{s}$, such that

$$
\sum_{i=1}^{s}\left(n_{i}+1\right)=n+1 .
$$

Using these data we can define an $S^{1}$-action $\varphi$ on $\mathbb{C} P^{n}$ by

$$
\begin{gathered}
\varphi: S^{1} \times \mathbb{C} P^{n} \longrightarrow \mathbb{C} P^{n}, \\
\left(z,\left[z_{0}, z_{1}, \ldots, z_{n}\right]\right) \longmapsto\left[z^{\lambda_{1}} z_{0}, \ldots, z^{\lambda_{1}} z_{n_{1}}, \ldots, z^{\lambda_{s}} z_{n-n_{s}}, \ldots, z^{\lambda_{s}} z_{n}\right],
\end{gathered}
$$

i.e., each $\lambda_{i}$ appears exactly $n_{i}+1$ times consecutively. Clearly

$$
\operatorname{Fix}\left(\mathbb{C} P^{n}\right)=\coprod_{i=1}^{s} \mathbb{C} P^{n_{i}}, \quad\left(\mathbb{C} P^{0}:=\{p t\}\right)
$$

and the weights of this action on the fixed point component $\mathbb{C} P^{n_{i}}$ are

$$
\left\{\lambda_{j}-\lambda_{i} \text { with multiplicity } n_{j}+1 \mid j \neq i\right\} .
$$

Thus, in $\mathrm{S}\left(\mathbb{C} P^{n}, \varphi\right)$, each $\lambda_{i}-\lambda_{j}(i \neq j)$ appears $\left(n_{i}+1\right)\left(n_{j}+1\right)$ times.

\section{Proof of Theorem 1.1}

We keep using the notation and symbols in Section 1. Now we recall the definition of

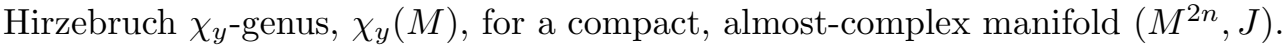

The choice of an almost Hermitian metric on $M$ enables us to define the Hodge star operator $*$ and the formal adjoint $\bar{\partial}^{*}=-* \bar{\partial} *$ of the $\bar{\partial}$-operator. Then for each $0 \leq p \leq n$, there is a Dolbeault-type elliptic operator

$$
\bigoplus_{q \text { even }} \Omega^{p, q}(M) \stackrel{\bar{\partial}+\bar{\partial}^{*}}{\longrightarrow} \bigoplus_{q \text { odd }} \Omega^{p, q}(M),
$$

where $\Omega^{p, q}(M):=\Gamma\left(\Lambda^{p} T^{*} M \otimes \Lambda^{q} \overline{T^{*} M}\right)$. Here $T^{*} M$ is the dual of holomorphic tangent bundle $T M$ in the sense of $J$. The index of this operator is denoted by $\chi^{p}(M)$. We define the Hirzebruch $\chi_{y}$-genus, $\chi_{y}(M)$, by

$$
\chi_{y}(M)=\sum_{p=0}^{n} \chi^{p}(M) \cdot y^{p} .
$$

Remark 2.1. (1) When $J$ is integrable, i.e., $M$ is an $n$-dimensional complex manifold, $\chi^{p}(M)$ is equal to the index of the following well-known Dolbeault complex

$$
0 \rightarrow \Omega^{p, 0}(M) \stackrel{\bar{\partial}}{\rightarrow} \Omega^{p, 1}(M) \stackrel{\bar{\partial}}{\rightarrow} \cdots \stackrel{\bar{\partial}}{\rightarrow} \Omega^{p, n}(M) \rightarrow 0
$$

and hence

$$
\chi^{p}(M)=\sum_{q=0}^{n}(-1)^{q} h^{p, q}(M),
$$

where $h^{p, q}(M)$ are the corresponding Hodge numbers of $M$. 
(2) It is a well-known fact that $\bar{\partial}^{2} \equiv 0$ is equivalent to the integrality of $J$. So for a general almost-complex manifold, $\bar{\partial}^{2}$ is not identically zero and thus we cannot define the Dolbeault complex (2.2). Therefore (2.1) can be viewed as the Dolbeault-type complex in the almost-complex case.

If we denote by $x_{i}, 1 \leq i \leq n$, the formal Chern roots of $T M$, i.e., the $i$-th elementary symmetric polynomial of $x_{1}, \ldots, x_{n}$ represents $c_{i}$, the $i$-th Chern class of $T M$, then the Atiyah-Singer Index Theorem tells us that

$$
\chi_{y}(M)=\prod_{i=1}^{n} \frac{x_{i}\left(1+y e^{-x_{i}}\right)}{1-e^{-x_{i}}} \cdot[M]
$$

where $[M]$ is the fundamental class of $M$ induced from $J$.

Moreover, if a compatible $S^{1}$-action $\varphi$ acts on $\left(M^{2 n}, J\right)$, then for any $g \in S^{1}$, we can define the corresponding equivariant indices $\chi^{p}(g, M)$ and equivariant Hirzebruch $\chi_{y}$-genus

$$
\chi_{y}(g, M):=\sum_{p=0}^{n} \chi^{p}(g, M) \cdot y^{p} .
$$

We still use $F$ to denote any connected component of the action $\varphi$ on $\left(M^{2 n}, J\right)$ with complex dimension $r$. Let $\alpha_{1}, \ldots, \alpha_{r}$ denote the formal Chern roots of $T F$, the holomorphic tangent bundle of $F$ in the sense of $\left.J\right|_{F}$. Let $t_{i}, 1 \leq i \leq n-r$, be the first Chern classes of the complex line bundles $\mathrm{L}\left(F, k_{i}\right)$. The following proposition is a generalization of $[5$, Theorem 2.2].

Proposition 2.2. Let the notation be as above. Then the following identity holds

$$
\chi_{y}(M) \equiv \sum_{F}\left[\left(\prod_{i=1}^{r} \alpha_{i} \frac{1+y e^{-\alpha_{i}}}{1-e^{-\alpha_{i}}}\right)\left(\prod_{j=1}^{n-r} \frac{1+y g^{k_{j}} e^{-t_{j}}}{1-g^{k_{j}} e^{-t_{j}}}\right)\right] \cdot[F],
$$

where $g$ is an indeterminate and the sum is over all the connected components in $\operatorname{Fix}(M)$.

Proof. The proof is similar to that of [5, Theorem 2.2] and the reader can also consult [4, p. 68-69]. For the sake of completeness, we still indicate it.

First, we choose a generic (topological generator) $g \in S^{1}$. Then the fixed point set of this $g$ is precisely the whole $\operatorname{Fix}(M)$. The Lefschetz fixed point formula of Atiyah-Bott-Segal-Singer [1, p. 562] tells us that

$$
\chi_{y}(g, M)=\sum_{F}\left[\left(\prod_{i=1}^{r} \alpha_{i} \frac{1+y e^{-\alpha_{i}}}{1-e^{-\alpha_{i}}}\right)\left(\prod_{j=1}^{n-r} \frac{1+y g^{k_{j}} e^{-t_{j}}}{1-g^{k_{j}} e^{-t_{j}}}\right)\right] \cdot[F] .
$$

Note that topological generators in $S^{1}$ are dense. Therefore (2.4) is an identity when taking $g$ as an indeterminate. Also note that the right-hand side (RHS) of (2.4) has well-defined limits at $g=0$ and $\infty$ as

$$
\begin{aligned}
& \lim _{g \rightarrow \infty}(\operatorname{RHS} \text { of }(2.4))=\sum_{F} \chi_{y}(F)(-y)^{d_{+}(F)}, \\
& \lim _{g \rightarrow 0}(\operatorname{RHS} \text { of }(2.4))=\sum_{F} \chi_{y}(F)(-y)^{d_{-}(F)},
\end{aligned}
$$


where $d_{+}(F)$ (resp. $d_{-}(F)$ ) is the number of positive (resp. negative) integers among $k_{1}, \ldots, k_{n-r}$. So $\chi_{y}(g, M)$ also has well-defined limits at $g=0$ and $\infty$. However, by definition $\chi_{y}(g, M)$ is a finite Laurent series of $g$, which means it must be a constant. Hence

$$
\chi_{y}(g, M) \equiv \chi_{y}(\mathrm{id}, M)=\chi_{y}(M), \quad \forall g .
$$

This completes the proof of this proposition.

We need the following lemma to derive Theorem 1.1.

Lemma 2.3. The Taylor expansion of

$$
\prod_{i=1}^{n} \frac{x_{i}\left(1+y e^{-x_{i}}\right)}{1-e^{-x_{i}}}
$$

at $y=-1$ is of the form

$$
c_{n}+\left(c_{n-1}-\frac{n}{2} c_{n}\right)(y+1)+\cdots .
$$

Consequently,

$$
\chi_{y}(M)=e(M)-\frac{n}{2} e(M)(y+1)+\cdots .
$$

Proof.

$$
\prod_{i=1}^{n} \frac{x_{i}\left(1+y e^{-x_{i}}\right)}{1-e^{-x_{i}}}=\prod_{i=1}^{n}\left[x_{i}+\frac{x_{i}}{e^{x_{i}}-1}(y+1)\right] .
$$

The coefficient of $y+1$ is

$$
\sum_{i=1}^{n}\left(\frac{x_{i}}{e^{x_{i}}-1} \cdot \prod_{j \neq i} x_{j}\right)=\sum_{i=1}^{n}\left[\left(1-\frac{1}{2} x_{i}\right) \cdot \prod_{j \neq i} x_{j}\right]=c_{n-1}-\frac{n}{2} c_{n} .
$$

Now we are in a position to prove Theorem 1.1.

Proof. From Lemma 2.3 we know

$$
\prod_{i=1}^{r} \frac{\alpha_{i}\left(1+y e^{-\alpha_{i}}\right)}{1-e^{-\alpha_{i}}}=c_{r}(F)+\left[c_{r-1}(F)-\frac{r}{2} c_{r}(F)\right] \cdot(y+1)+\cdots,
$$

and

$$
\begin{aligned}
\prod_{j=1}^{n-r} \frac{1+y g^{k_{j}} e^{-t_{j}}}{1-g^{k_{j}} e^{-t_{j}}} & =\prod_{j=1}^{n-r}\left[1+\frac{g^{k_{j}} e^{-t_{j}}}{1-g^{k_{j}} e^{-t_{j}}}(y+1)\right] \\
& =1+\sum_{j=1}^{n-r} \frac{g^{k_{j}} e^{-t_{j}}}{1-g^{k_{j}} e^{-t_{j}}}(y+1)+\cdots \\
& =1+\left(\sum_{j=1}^{n-r} \frac{g^{k_{j}}}{1-g^{k_{j}}}+\text { higher degree terms }\right) \cdot(y+1)+\cdots
\end{aligned}
$$


Using (2.5), (2.6), and comparing the coefficients of $y+1$ on both sides of (2.3), we deduce that

$$
\begin{aligned}
-\frac{n}{2} e(M) & =\sum_{F}\left[-\frac{r}{2} e(F)+e(F) \sum_{j=1}^{n-r} \frac{g^{k_{j}}}{1-g^{k_{j}}}\right] \\
& =\sum_{F}\left[\left(\frac{r}{2}-n\right) e(F)+e(F) \sum_{j=1}^{n-r} \frac{1}{1-g^{k_{j}}}\right] .
\end{aligned}
$$

Letting $y=-1$ in (2.3) we have the well-known identity

$$
e(M)=\sum_{F} e(F) .
$$

Using this identity to substitute $e(M)$ in $(2.7)$, we obtain

$$
\begin{aligned}
\frac{1}{2} \sum_{F}(n-r) e(F)= & \sum_{F}\left[e(F) \sum_{j=1}^{n-r} \frac{1}{1-g^{k_{j}}}\right] \\
= & \sum_{e(F)>0}\left[e(F) \sum_{j=1}^{n-r} \frac{1}{1-g^{k_{j}}}\right] \\
& +\sum_{e(F)<0}\left[-e(F) \sum_{j=1}^{n-r}\left(\frac{1}{1-g^{-k_{j}}}-1\right)\right] .
\end{aligned}
$$

Rewriting (2.8), we have

$$
\sum_{e(F)>0}\left[e(F) \sum_{j=1}^{n-r} \frac{1}{1-g^{k_{j}}}\right]+\sum_{e(F)<0}\left[-e(F) \sum_{j=1}^{n-r} \frac{1}{1-g^{-k_{j}}}\right]=\frac{1}{2} \sum_{F}(n-r)|e(F)| .
$$

Then Theorem 1.1 follows from the following lemma, which should be well known and has been used implicitly in the proof of Theorem 3.5 in [5]. Unfortunately we cannot find a reference for this lemma. To make our proof more rigorous, we give a detailed proof of this lemma ourselves.

Lemma 2.4. Suppose we have $m$ non-zero integers $k_{1}, \ldots, k_{m}$, which are not necessarily distinct, and an indeterminate $g$. Then as a rational function,

$$
\sum_{i=1}^{m} \frac{1}{1-g^{k_{i}}} \equiv \text { constant }
$$

if and only if $m$ is even, constant $=\frac{m}{2}$, and there exist $\frac{m}{2}$ positive integers $p_{1}, \ldots, p_{\frac{m}{2}}$, which are not necessarily distinct either, such that

$$
\left\{k_{1}, \ldots, k_{m}\right\}=\left\{p_{1}, \ldots, p_{\frac{m}{2}}\right\} \cup\left\{-p_{1}, \ldots,-p_{\frac{m}{2}}\right\} .
$$

Proof. The "if" part is trivial as

$$
\frac{1}{1-g^{k}}+\frac{1}{1-g^{-k}} \equiv 1
$$

for any non-zero integer $k$. 
Now we prove the "only if" part. Assume that there are $r$ negative integers among $k_{1}, \ldots, k_{m}$.

$$
\sum_{i=1}^{m} \frac{1}{1-g^{k_{i}}}=\sum_{k_{i}>0} \frac{1}{1-g^{k_{i}}}+\sum_{k_{i}<0}\left(1-\frac{1}{1-g^{-k_{i}}}\right) \equiv \text { constant }
$$

which implies

$$
\sum_{k_{i}>0} \frac{1}{1-g^{k_{i}}}=\text { constant }-r+\sum_{k_{i}<0} \frac{1}{1-g^{-k_{i}}} .
$$

Letting $g$ tend to 0 and $\infty$ on both sides of (2.9), we have

$$
m-r=\text { constant }, \quad 0=\text { constant }-r .
$$

Thus $m$ is even, constant $=\frac{m}{2}$, and (2.9) becomes

$$
\sum_{k_{i}>0} \frac{1}{1-g^{k_{i}}}=\sum_{k_{i}<0} \frac{1}{1-g^{-k_{i}}} .
$$

Now assume that $g$ is a complex variable with $|g|<1$. We consider the Taylor expansion on both sides of (2.10)

$$
\sum_{k_{i}>0}\left(g^{k_{i}}+g^{2 k_{i}}+\cdots\right)=\sum_{k_{i}<0}\left(g^{-k_{i}}+g^{-2 k_{i}}+\cdots\right) .
$$

Comparing the lowest order terms of $g$ on both sides of (2.11) step by step for $\frac{m}{2}$ times, we deduce that

$$
\left\{k_{i} \mid k_{i}>0\right\}=\left\{-k_{i} \mid k_{i}<0\right\} .
$$

This completes the proof of Lemma 2.4 and thus Theorem 1.1.

Remark 2.5. Although we state this lemma for integers $k_{i}$, from the proof we can easily see that this lemma still holds if we replace these integers with real numbers.

\section{Concluding remarks}

Several articles have noted that, implicitly or explicitly, at $y=-1$, the coefficients of lower order terms in the Taylor expansion of $\chi_{y}(M)$ could be given explicit expressions and many applications. More precisely, if we write

$$
\chi_{y}(M)=: \sum_{i=0}^{n} a_{i}(M) \cdot(1+y)^{i}
$$

then

$$
a_{0}=c_{n}[M], \quad a_{1}=-\frac{n}{2} c_{n}[M], \quad a_{2}=\frac{1}{12}\left[\frac{n(3 n-5)}{2} c_{n}+c_{1} c_{n-1}\right][M] .
$$

The calculation of $a_{0}$ is trivial as we can set $y=-1$ in $\chi_{y}(M)$. The calculation of $a_{1}$ has been done in our Lemma 2.3. The calculation of $a_{2}$ appears implicitly in [8, p. 18] and [2, Corollary 5.3.12] and explicitly in [7, p. 141-143]. Narasimhan and Ramanan used $a_{2}$ to give a topological restriction on some moduli spaces of stable vector bundles over Riemann surfaces. The primary interest of [2] in chapter five is to interpret the famous Futaki invariant of Fano manifolds as a special case of a family of integral invariants. However in Corollary 5.3.12, Futaki also implicitly 
computed the expression $a_{2}$. Libgober and Wood used $a_{2}$ to prove the uniqueness of the complex structure on Kähler manifolds of certain homotopy types [7, Theorems 1 and 2]. Inspired by Narasimhan-Ramanan's article [8], Salamon applied $a_{2}$ [10, Corollary 3.4] to obtain a restriction on the Betti numbers of hyper-Kähler manifolds [10, Theorem 4.1]. In [3], Hirzebruch used $a_{1}, a_{2}$ and $a_{3}$ to deduce a divisibility result on the Euler number of almost-complex manifolds with $c_{1}=0$. In [6, p. 182], the author also provided a method to calculate $a_{2}$.

Keeping these backgrounds in mind, the starting point of this paper is to compare $a_{0}, a_{1}$ and $a_{2}$ on both sides of (2.3) to see what we can obtain. Comparing $a_{0}$ leads to

$$
e(M)=\sum_{F} e(F)
$$

which is well known and has been used in our proof of Theorem 1.1. Comparing $a_{1}$ leads to our main result in this note. One may ask what we can obtain if we compare $a_{2}$ on both sides of (2.3). Unfortunately in general we cannot get useful information even in the case that $\operatorname{Fix}(M)$ is isolated, which we shall explain in what follows.

Suppose the fixed points are isolated, say $\left\{P_{1}, \ldots, P_{r}\right\}$, and at each $P_{i}$ the weights are $k_{1}^{(i)}, \ldots, k_{n}^{(i)}$. Then $(2.3)$ reduces to

$$
\chi_{y}(M)=\sum_{i=1}^{r} \prod_{j=1}^{n} \frac{1+y g^{k_{j}^{(i)}}}{1-g^{k_{j}^{(i)}}}=\sum_{i=1}^{r} \prod_{j=1}^{n}\left[1+\frac{g^{k_{j}^{(i)}}}{1-g^{k_{j}^{(i)}}}(1+y)\right],
$$

which is exactly what [5, Theorem 2.2] considers. Comparing $a_{2}(M)$ on both sides of (3.1), we have

$$
\frac{1}{12}\left[\frac{n(3 n-5)}{2} c_{n}+c_{1} c_{n-1}\right][M]=\sum_{i=1}^{r} \sum_{1 \leq p<q \leq n} \frac{g^{k_{p}^{(i)}+k_{q}^{(i)}}}{\left(1-g^{k_{p}^{(i)}}\right)\left(1-g^{k_{q}^{(i)}}\right)} .
$$

The expression of the RHS of (3.2) is closely related to an old result of Zagier [11], which in turn is inspired by a conjecture connected with the topology of threedimensional manifolds. This conjecture asserted that the rational functions

$$
f_{r s}(g)=\frac{\left(1+g^{r}\right)\left(1+g^{s}\right)}{\left(1-g^{r}\right)\left(1-g^{s}\right)} \quad(r \geq 2 s>0)
$$

are linearly independent over the integers. For the topological background of this conjecture, we refer the readers to [11, p. 321]. In [11] Zagier showed that there are in fact infinitely many linear relations among these rational functions $f_{r s}(g)$ and hence disproved this conjecture [11, p. 322]. Therefore through Zagier's this result we cannot expect that, for these weights $k_{j}^{(i)}$ satisfying (3.2), there is an analogue to Lemma 2.4 when considering $a_{2}$.

\section{Acknowledgments}

The paper was completed when the author was holding a JSPS Postdoctoral Fellowship in the Department of Mathematics at Tokyo Metropolitan University. The author thanks the Department and Professor Martin Guest for their hospitality. Special thanks should also go to the referee, whose many fruitful suggestions and comments improve the quality of this paper. This work was partially supported by the 
National Natural Science Foundation of China (grant no. 11101308) and the JSPS Postdoctoral Fellowship for Foreign Researchers.

\section{References}

[1] M.F. Atiyah and I.M. Singer, The index theory of elliptic operators: III, Ann. Math. 87 (1968), 546-604.

[2] A. Futaki, Kähler-Einstein metrics and integral invariants, Lecture Notes in Mathematics, 1314, Springer-Verlag, Berlin, 1988.

[3] F. Hirzebruch, On the Euler characteristic of manifolds with $c_{1}=0$. A letter to V. Gritsenko, St. Petersburg Math. J. 11 (2000), 805-807.

[4] F. Hirzebruch, T. Berger and R. Jung, Manifolds and modular forms, Aspects of Mathematics, E20, Friedr. Vieweg and Sohn, Braunschweig, 1992.

[5] P. Li, The rigidity of Dolbeault-type operators and symplectic circle actions, Proc. Amer. Math. Soc. 140 (2012), 1987-1995.

[6] P. Li, Chern numbers and the indices of some elliptic differential operators, Pacific J. Math. 251 (2011), 173-182.

[7] A. Libgober and J. Wood, Uniqueness of the complex structure on Kähler manifolds of certain homology types, J. Differ. Geom. 30 (1990), 139-154.

[8] M.S. Narasimhan and S. Ramanan, Generalized Prym varieties as fixed points, J. Ind. Math. Soc. 39 (1979), 1-19.

[9] A. Pelayo and S. Tolman, Fixed points of symplectic periodic flows, Ergodic Theory Dyn. Syst. 31 (2011), 1237-1247.

[10] S.M. Salamon, On the cohomology of Kähler and hyper-Kähler manifolds, Topology 35 (1996), 137-155.

[11] D. Zagier, Relations among invariants of circle actions on three-manifolds, Math. Ann. 195 (1972), 321-329.

Department of Mathematics, Tongui University, Shanghai 200092, People's Republic OF CHINA

E-mail address: pingli@tongji.edu.cn 
